\title{
LYVE-1 expression in liver cells of mice with functional pinealectomy
}

\author{
Irina Yurievna Ishchenko \\ Group of experimental \\ pharmacology \\ RICEL - Branch of ICG SB RAS \\ Novosibirsk, Russia \\ irenisch@mail.ru
}

\author{
Andrey Yurievich Letyagin \\ Laboratory of pharmaceutical \\ technologies \\ RICEL - Branch of ICG SB RAS \\ Novosibirsk, Russia \\ letyagin-andrey@yandex.ru
}

\author{
Svetlana Viktorovna Michurina \\ Group of experimental \\ pharmacology \\ RICEL - Branch of ICG SB RAS \\ Novosibirsk, Russia \\ s.michurina@ngs.ru
}

\author{
Maxim Aleksandrovich Korolev \\ Laboratory of connective tissue \\ pathology \\ RICEL - Branch of ICG SB RAS \\ Novosibirsk, Russia \\ kormax@bk.ru
}

\author{
Sergey Alekseevich Arkhipov \\ Group of experimental \\ pharmacology \\ RICEL - Branch of ICG SB RAS \\ Novosibirsk, Russia \\ arhipowsergei@yandex.ru
}

\author{
Evgenii Leonidovich Zavjalov \\ Center of genetis resources of \\ laboratory animals \\ ICG SB RAS \\ Novosibirsk, Russia \\ zavjalov@bionet.nsc.ru
}

\begin{abstract}
The expression of lymphatic vessel endothelial hyaluronan receptor-1 (LYVE-1) in liver cells of C57BL/6 mice males kept 14 days under 24-hour lighting (mice with functional pinealectomy) was studied. The immunohistochemical analysis (indirect avidin-biotin ABC-peroxidase method) and morphometric assessment were used. A 2 -fold decrease in the area of LIVE-1 expression was found against the background of a $2 \%$ increase in the optical density of LIVE-1 staining in mouse liver cells after chronic continuous illumination. The weak LYVE-1 expression on the membranes of endothelial cells of liver sinusoids may indicate a violation of the functioning of the fenestration fields of these cells. This can lead to a decrease in the endocytotic activity of the latter, difficulty in hemato-tissue exchange, lymphatic drainage deterioration and tissue hypoxia development in the liver of mice with functional pinealectomy.
\end{abstract}

Key words - continuous illumination, functional pinealectomy, liver, LIVE-1

\section{Introduction}

An integral factor of modern life is the use of continuous artificial lighting and constant changes in light mode. As a result of the long existence of a person under such conditions, the production of the pineal gland hormone melatonin significantly decreases/stops in his body (functional pinealectomy), there is an imbalance of cyclicity in the work of body systems, desynchronosis develops.

It is known that the liver is not only a unique metabolic and immunological organ, it is the largest source of lymph production in the body, and it accounts for up to $50 \%$ of the lymph entering the thoracic duct [1]. The lymphatic system of any organ helps maintain tissue fluid balance and remove cellular waste products. It was found that with liver diseases its lymphatic system changes significantly. An increase in hepatic lymphatic vessels with cirrhosis, viral hepatitis and hepatocellular carcinoma has been reported [2]. It was found that under the influence of constant lighting in the liver and its lymphatic region, circulatory and lymphatic flow disorders develop. These processes lead to the development of hypoxia, which negatively affects the structure and function of the organ parenchyma cells [3]. The aim of this study was to evaluate the effect of 24-hour illumination $(24 \mathrm{hL})$ on the expression of the LIVE-1 marker (lymphatic vessel endothelial receptor-1) in liver cells of male C57B1 / 6 mice.

\section{Materials and Methods}

The males of $\mathrm{C} 57 \mathrm{Bl} / 6$ mice aged of $10-12$ weeks were kept in the SPF-vivarium center of ICG SB RAS. Food and water were provided to animals at libitum. The intact animals (the group "Control", $n=6$ ) were kept under the standard lighting mode - photoperiod light/dark: 14/10 hour (h). The mice of the experimental group (the gr. "24hL", n=6) were kept for 14 days under conditions of 24-hour lighting - photoperiod light/dark: 24/0 h. Animals were removed from the experiment by the cranio-cervical dislocation method and liver samples were taken for light-optical and immunohistochemical studies.Liver samples were fixed in $10 \%$ buffered formalin for 48 hours, dehydrated in a series of alcohols of increasing concentrations and embedded in Histomix. A light-optical study of the liver was performed on sections stained with Mayer hematoxylin and eosin.

Immunohistochemical detection of the LYVE-1 marker was performed in sections with a $3 \mu \mathrm{m}$ thickness using the indirect ABC-peroxidase method using the VECTASTAIN Universal ABC-Peroxidase Kit (PK-7200, Vector Laboratories, USA). Endogenous peroxidase blocking was performed by incubation of sections in $0.3 \% \mathrm{H}_{2} \mathrm{O}_{2}$ solution for $10 \mathrm{~min}$, then sections were incubated with normal blocking non-immune horse serum for $20 \mathrm{~min}$. Next, the sections were incubated for an hour at room temperature with antibodies to LYVE-1 (Isotype: Rabbit polyclonal, bs-1311R; Bioss) using the final dilution of $5 \mu \mathrm{g} / \mathrm{ml}$, then they were washed in 3 shifts of phosphate buffer (FB) for $3 \mathrm{~min}$ and were incubated for 30 minutes at room temperature with the second biotinylated antibodies followed by washing in 3 shifts of FB for 5 minutes. Incubation with the ABC-peroxidase complex was carried out at room temperature for $30 \mathrm{~min}$, the sections were washed in 3 shifts of $\mathrm{FB}$ for 5 minutes. At the last stage immunohistochemical staining of the sections was performed in the chromogenic substrate (ImmPACT DAB, Vector Laboratories, USA). For quantification of LIVE-1 expression in the mouse liver, there was performed computer morphometric analysis of digital photographs at $\times 400$ magnification. The relative areas and optical densities of 
zones staining for LIVE-1 in digital images were determined using the program "Image J". A comparison of the results between the groups "24hL" and "Control" was performed using the Mann-Whitney U-test for abnormally distributed characters in independent samples. Differences of compared values were considered statistically significant at $\mathrm{P}<0.05$.

\section{Results and Discussion}

In the liver of mice after two weeks of round-the-clock lighting $(24 / 0 \mathrm{~h})$, dilatation of the spaces of the Malla and lymphatic vessels, expansion of the bile ducts in the portal tracts were detected. These changes were accompanied by edema of the interlobular connective tissue layers. There was a stasis of red blood cells in the interlobular, central and sublobular veins. The intralobular sinusoid capillaries were also expanded. Lymphoid nodules were found in the parenchyma.

The study of the expression of the LIVE-1 marker in the liver of intact mice under standard lighting mode revealed a pronounced immunohistochemical staining of sinusoid cells of blood capillaries in the periportal regions and especially in the perineal zones of the hepatic lobes. In the parenchyma, individually colored hepatocytes were sometimes found. In animals kept under round- the-clock lighting, a weaker expression of LIVE-1 was detected in the endothelium of sinusoid capillaries, especially in the intermediate zones . Morphometric analysis of mouse liver preparations after two weeks of continuous illumination revealed a two-fold decrease in the area of expression of the LIVE-1 marker against the background of an increase in its optical density of LIVE-1 staining by $2 \%$.

LYVE-1 is known to be a transmembrane protein receptor in endothelial cells of lymphatic vessels, for hyaluronic acid( hyaluronan), an extracellular matrix mucopolysaccharide [4]. Hyaluronan is the most powerful water-binding molecule in our body; it can absorb up to 1000 times its mass in water [5]. The pronounced expression of LIVE-1 in the endothelium of liver sinusoids in intact mice is confirmed by the results of other researchers. It was found that LYVE-1 is expressed in the liver not only by endothelial cells of lymphatic vessels but also by endothelial cells of blood sinusoid capillaries, as well as in some macrophages. As in our study, the primary staining on LIVE-1 of portal and especially middle zones of liver acini in intact animals was previously revealed. It is known that parenchyma cells in the intermediate zones are most sensitive to oxidative stress [6].

In liver diseases, the expression of LIVE-1 may be reduced. Thus, in cirrhosis and hepatocellular carcinoma, LYVE-1 expression is suppressed [6]. In chronic hepatitis and cirrhosis of the liver, a decrease in the expression of LYVE-1 in the endothelium of sinusoids was detected, especially in areas adjacent to active inflammatory or fibrotic lesions. In this case, the sinusoidal endothelium in the affected areas, demonstrating the weakening of LYVE-1, loses fenestration, which the authors attribute to the progression of liver disease [7]. Disorders in the lymphatic vasculature that cause inadequate drainage can lead to a condition characterized by tissue swelling, which is the result of the accumulation of protein and fluid in the interstitium [8].

In this regard, the weak expression of LYVE-1 detected by us on the membranes of endothelial cells of sinusoidal capillaries in mice kept for a long time under continuous illumination may indicate a violation of the functioning of the fields of fenestration of these cells. This can lead to a decrease in the endocytotic activity of the latter, difficulty in bloodtissue exchange, deterioration of lymphatic drainage in the liver, and development of tissue hypoxia.

\section{ACKNOWLEDGMENT}

The study is supported by the budget project (No. AAAAA17-17072710029-7) and implemented using the equipment of the Center for Genetic Resources of Laboratory Animals at ICG SB RAS, supported by the Ministry of Education and Science of Russia (Unique identifier of the project RFMEFI62119X0023).

\section{REFERENCES}

[1] M. Tanaka and Y. Iwakiri, "The Hepatic Lymphatic Vascular System: Structure, Function, Markers, and Lymphangiogenesis," Cell. Mol. Gastroenterol. Hepatol., vol. 2, № 6, pp. 733-749, 2016.

[2] M. Tanaka and Y. Iwakiri, "Lymphatics in the liver," Curr. Opin. Immunol., vol. 53, pp. 137-142, 2018.

[3] S. V. Michurina, Yu. I Borodin., I. Yu. Ishchenko, A.D. Belkin, A.V. Shurlygina and P.M. Larionov, "The lymphatic region of Wistar rat liver under conditions of combined influence of alcohol intoxication and round-the-clock lighting," Bulletin of SB RAMS, vol. 5, pp. 44-49, 2008. (in Russian).

[4] J.W. Breslin, Y. Yang, J.P. Scallan, R.S. Sweat, S.P. Adderley and W.L. Murfee, "Lymphatic Vessel Network Structure and Physiology," Compr. Physiol., vol. 9, № 1, pp. 207-299, 2018.

[5] A.J. Day and J.K Sheehan, "Hyaluronan: polysaccharide chaos to protein organisation,” Curr. Opin. Struct. Biol., vol. 11, № 5, pp. 617622, 2001.

[6] C. Carreira Mouta, S.M. Nasser, E. di Tomaso, T.P. Padera, Y. Boucher, S.I. Tomarev and R.K. Jain, "LYVE-1 is not restricted to the lymph vessels: expression in normal liver blood sinusoids and downregulation in human liver cancer and cirrhosis," Cancer Res., vol. 61, № 22, pp.8079-8084, 2001.

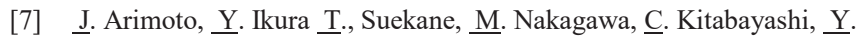
Iwasa, K. Sugioka, T. Naruko, T. Arakawa and Ueda M., "Expression of LYVE-1 in sinusoidal endothelium is reduced in chronically inflamed human livers,” J. Gastroenterol., vol. 45, № 3, pp. 317-325, 2010.

[8] P. Saharinen, T. Tammela, M.J. Karkkainen and K. Alitalo, "Lymphatic vasculature: development, molecular regulation and role in tumor metastasis and inflammation,” Trends Immunol., vol. 25, № 7, pp.387-395, 2004. 